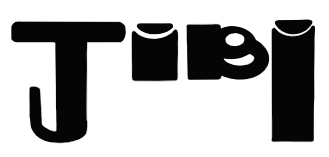

JORNAL INTERDISCIPLINAR DE BIOCIÊNCIAS

Homepage: http://www.ojs.ufpi.br/index.php/jibi

\title{
Forame Mentual: verificação da localização por meio de radiografia panorâmica
}

\section{Mental Foramen: location verification by means of panoramic radiography}

Ingrid Macedo de Oliveira ${ }^{1}$, Samuel Oliveira Menezes ${ }^{2}$, Carlos Alberto Monteiro Falcão ${ }^{2}$, Maria Ângela Arêa Leão ${ }^{2}$, Márcia dos Santos Rizzo ${ }^{1}$, Aírton Mendes Conde Junior ${ }^{1}$, Carla Maria Carvalho Leite $^{1 *}$

${ }^{1}$ Departamento de Morfologia, Universidade Federal do Piaui; ${ }^{2}$ Centro Universitário Uninovafapi

\begin{abstract}
A B S TR A C T
The topography of the mentual foramen is of great importance in the dental clinic in order to provide the performance of safe procedures in the mandible. However, their location and characteristics may vary among populations. The objective of the present study was to describe the topography and anatomical variations of the mental foramen by means of panoramic radiographs. A retrospective cross-sectional study, performed by a previously calibrated examiner, analyzed the right and left antigens of 100 panoramic radiographs, totaling 200 foramens.. Mental foramen was classified according to the position in relation to the lower teeth and the height relative to the base of the mandible. The radiographs were analyzed using a negatoscope and a digital caliper. As for the position of the teeth, it was observed that the class III foramen (62\%) was the one with the highest prevalence, followed by class VI (23.5\%), class V (10.5\%), class VI (2\%) and classes I (1\%) and II (1\%). The highest heights were $13 \mathrm{~mm}$ and $14 \mathrm{~mm}$ for the left and right sides respectively. There were variations in the localization of the mental foramen, with a higher prevalence of the foramen situated between the first and second premolars. However, there was proximity between the dimensions and the location of the mental foramen on both sides of the same radiographic examination.
\end{abstract}

KEYWORDS

Mandible, anatomic variation, panoramic radiograph

RES U M O

A topografia do forame mentual é de grande importância na clínica odontológica, porque proporciona a realização de procedimentos seguros na mandíbula; no entanto, sua localização e características podem variar entre as populações. O objetivo do presente estudo foi descrever a topografia e as variações anatômicas do forame mental por meio de radiografias panorâmicas. Sendo um estudo retrospectivo transversal, feito por examinador previamente calibrado, foram analisados os antímeros direito e esquerdo de 100 radiografias panorâmicas, totalizando 200 forames. $\mathrm{O}$ forame foi classificado quanto à posição, em relação aos dentes inferiores, e quanto à altura, em relação à base da mandíbula. A análise das radiografias foi realizada sob negatoscópio e com auxílio de paquímetro digital. A estatística foi do tipo descritiva. Quanto à posição dos dentes, observou-se que o forame classe III (62\%) foi o de maior prevalência, seguido pelos de classe IV (23,5\%), classe V (10,5\%), classe VI (2\%) e classes I (1\%) e II (1\%). As maiores alturas encontradas foram de $13 \mathrm{~mm}$ e $14 \mathrm{~mm}$ para os lados esquerdo e direito respectivamente. Houve variações na localização do forame mental, com maior prevalência do forame situado entre o primeiro e o segundo pré-molar. Porém houve proximidade entre as dimensões e a localização do forame mental nos dois lados de um mesmo exame radiográfico.

PA L A VRAS - CHAVE

Mandíbula, variação anatômica, radiografia panorâmica

\section{INTRODUÇÃO}

O conhecimento anatômico do forame mentual (FM) e suas variações são de interesse em diversas áreas da odontologia. Durante procedimentos cirúrgicos, como cirurgias ortognáticas e instalação de implantes (BAHLIS et al., 2010) e até mesmo durante a anestesia, o AUTOR CORRESPONDENTE

Ingrid Macedo de Oliveira < ingridmacedo13@hotmail.com >

Departamento de Morfologia - Universidade Federal do Piauí

Campus Ministro Petrônio Portela - Bairro: Ininga -- CEP: 64049-550

Teresima - PI planejamento anatômico é imprescindível, evitando danos ao feixe vásculo-nervoso presente na região (TEIXEIRA et al., 2007; MORAES et al., 2008; MANHÃES et al., 2009; LIMA et al., 2010). Ademais, a distinção entre processos patológicos e estruturas anatômicas na mandíbula torna-se, muitas vezes, difícil durante clínica, assim como no 
diagnóstico diferencial entre lesões periapicais e o FM (RIBEIRO, FERREIRA, 2015).

$\mathrm{O}$ forame mentual corresponde à abertura do canal mandibular presente na cortical vestibular do corpo da mandíbula, onde emerge o feixe vásculo-nervoso mentoniano. Há pouca concordância sobre sua exata localização (MORAES et al., 2008; MANHÃES et al., 2009; GUEDES et al., 2011). Estudos mostram que o forame pode variar quanto à altura em relação à base da mandíbula, à posição em relação aos dentes inferiores e à sua forma, oval ou arredondada (HAGHANIFAR; ROKOUEI, 2009; GUEDES et al., 2011). Além dessas características, o FM pode ser variável entre as populações (NEIVA et al., 2004; LIMA et al., 2010; SANKAR; BHANU; SUSAN, 2011), justificando a realização de novos estudos.

A radiografia panorâmica é considerada um exame de triagem básico de grande importância, podendo auxiliar até mesmo no diagnóstico da osteoporose (BAHLIS, 2010; MANHÃS et al., 2009; FILHO et al., 2011; Leite et al., 2008). Apesar das limitações inerentes à própria técnica envolvendo a distorção de imagens (COMANDULLI et al., 2005; SALVADOR et al., 2010) deve ser um exame de rotina na clínica odontológica, pois permite uma visualização geral do terço inferior da face a um baixo custo (ANDRADE et al., 2015).

O conhecimento da topografia e características do forame mentual é de grande importância na prática clínica, uma vez que evita danos ao feixe vásculo-nervoso referente e um diagnóstico errôneo de patologia. A radiografia panorâmica é um recurso chave e de baixo custo na identificação de estruturas anatômicas e alterações na mandíbula. Assim, esta pesquisa proporciona a identificação das características do FM em uma determinada população. O presente estudo propõe, então, a descrição da topografia e as variações anatômicas do forame mentual por meio de radiografias panorâmicas.

\section{MATERIAIS E MÉTODOS}

Este estudo trata-se de uma pesquisa retrospectiva transversal, realizada após análise e aprovação do Comitê de Ética e Pesquisa do UNINOVAFAPI (parecer $\mathrm{n}^{\circ}$ 261.084), conforme a resolução 466/12 CNS/CONEP, que versa sobre a realização de pesquisas com seres humanos. Enquadrado como uma investigação de risco mínimo, foi necessário assinatura do termo de fiel depositário, por parte da instituição de ensino, que cedeu as radiografias, além do uso do termo de confidencialidade por parte dos pesquisadores.

Avaliou-se o forame mentual por meio de radiografias panorâmicas. Utilizou-se 100 radiografias panorâmicas de pacientes dos gêneros masculino e feminino, atendidos na
Clínica Integrada de Saúde do Centro Universitário UNINOVAFAPI, Teresina-PI, Brasil, que demandaram serviços odontológicos e realizaram radiografias panorâmicas no período de 2005 a 2011. Foram eleitas as radiografias com adequados padrões técnicos e pertencentes a prontuários devidamente preenchidos. Os critérios de exclusão foram radiografias de pacientes com ausências de dentes posteriores e desdentados totais. A amostra foi do tipo censitário, todas as 100 radiografias presentes no acervo da instituição, durante o período de realização do estudo, foram utilizadas.

A análise dos exames radiográficos foi realizada por um único examinador previamente treinado, com a supervisão do orientador. As interpretações foram realizadas em ambiente com iluminação reduzida e as imagens foram observadas em negatoscópio, recoberto com máscara de proteção preta, para eliminar o excesso de luz. As radiografias foram fixadas e interpretadas com o auxílio de lupa (aumento $=2 \mathrm{X}$ ). Cada lado da mandíbula foi observado separadamente. A topografia do forame mentual foi verificada de acordo com a classificação proposta por Tebo, Telford (1950) (Tabela 1).

\section{Tebo e Telford (1950)}

Tipo I - Forame mentual localizado entre os caninos inferiores e os primeiros pré-molares inferiores.

Tipo II - Forame mentual localizado abaixo dos primeiros pré-molares inferiores.

Tipo III - Forame mentual localizado entre os primeiros e segundos pré-molares inferiores.

Tipo IV - Forame mentual localizado abaixo dos segundos pré-molares inferiores.

Tipo V - Forame mentual localizado entre os segundos pré-molares inferiores e os primeiros molares inferiores.

Tipo VI - Forame mentual localizado abaixo dos primeiros molares inferiores.

Tabela 1. Classificação do forame mentual proposta por Tebo e Telford (1950). Fonte: Tebo e Telford (1950).

Constatou-se a topografia do FM em relação aos ápices dos dentes caninos, pré molares e molares, e a base da mandíbula, identificando a prevalência de variações anatômicas associadas ao gênero. Os dados foram anotados em ficha própria, confeccionada pelo orientador para este fim.

As medições foram realizadas com paquímetro digital (King Tools, Resolução de 0,01mm, São Paulo, Brasil) e régua milimétrica, além de distribuídas em tabelas. Os dados foram analisados por meio do programa estatístico SPSS (Statistical Package for the Social Sciences ${ }^{\circledR}$, Versão 20.0, Chicago, EUA) em estatística descritiva. 


\section{RESULTADOS E DISCUSSÃO}

O forame mentual é um importante ponto anatômico situado na face vestibular do corpo da mandíbula. Sua localização influência planejamentos cirúrgicos realizados em diversas especialidades da odontologia (BAHLIS et al., 2010). No entanto, pode haver variações quanto à sua posição, características e entre as populações (TEBO, TELFORD, 1950; NEIVA et al., 2004; LIMA et al., 2010). Neste estudo, analisou-se a topografia do FM por meio de radiografias panorâmicas. A amostra foi de 100 radiografias, nas quais $61 \%$ foram de pacientes do gênero feminino e $39 \%$ do gênero masculino.

Estudiosos consideram que a etnia, gênero e idade são fatores responsáveis pela variação topográfica do forame mentual (APINHASMIT et al., 2006; HAGHANIFAR, ROKOUEI, 2009). Observou-se, neste estudo, a variabilidade no posicionamento do FM em relação aos ápices radiculares (Tabela 2), assim como citado na literatura (FILHO et al., 2011; GUEDES et al., 2011).

Quanto à posição em relação aos ápices radiculares, a maior prevalência foi de forame mentual classe III (62\%), entre os primeiros e os segundos pré-molares inferiores, sendo 64\% no antímero direito e $60 \%$ no esquerdo (Tabela 2). Dados que corroboram com os estudos de Manhães Jr et al. (2009) que verificaram essa posição em $71 \%$ dos casos, Guedes et al. (2011) em 55,89\% e Filho et al. (2011) em 54\%. Resultados semelhantes também foram observados por Araújo et al. (2005) em 72,3\% e Teixeira et al. (2007) em 70,78\% dos casos; ambos, porém, estudaram o forame em radiografias periapicais.

Estudos realizados em diferentes grupos populacionais mostram que a localização do FM é predominantemente, abaixo do segundo pré-molar classe IV (APINHASMIT et al, 2006; KIM et al, 2006; GUO et al, 2009, SANKAR, BHANU, SUSAN, 2011; SAITO, et al., 2015). No pre-

\begin{tabular}{ccccc}
\hline & \multicolumn{1}{c}{$\begin{array}{c}\text { Antímero } \\
\text { direito }\end{array}$} & $\begin{array}{c}\text { Antímero } \\
\text { esquerdo }\end{array}$ & $\begin{array}{c}\text { Antímeros direito e } \\
\text { esquerdo }\end{array}$ \\
\hline & Tipo I & $1(1 \%)$ & $1(1 \%)$ & $2(1 \%)$ \\
\cline { 2 - 5 } & Tipo II & 0 & $2(2 \%)$ & $2(1 \%)$ \\
\cline { 2 - 5 } $\begin{array}{c}\text { Tipo III } \\
\text { Tebsificação de }\end{array}$ & $64(64 \%)$ & $60(60 \%)$ & $124(62 \%)$ \\
\cline { 2 - 5 } & Tipo IV & $24(24 \%)$ & $23(23 \%)$ & $47(23,5 \%)$ \\
\cline { 2 - 5 } & Tipo V & $9(9 \%)$ & $12(12 \%)$ & $21(10,5 \%)$ \\
\cline { 2 - 5 } & Tipo VI & $2(2 \%)$ & $2(2 \%)$ & $4(2 \%)$ \\
\cline { 2 - 5 } & Total & $100(50 \%)$ & $100(50 \%)$ & $200(100 \%)$ \\
\hline
\end{tabular}

Tabela 2. Classificação topográfica do forame mentual em relação ao ápice dos dentes caninos, pré-molares e molares segundo a classificação de Tebo e Telford (1950) nos antímeros direito e esquerdo. Fonte: Acervo de Radiografias do UNINOVAFAPI, Teresina-PI.

-sente estudo, o FM esteve a baixo do segundo pré-molar em 23,5\% dos casos (Tabela 2), assim como verificado por Morais et al. 2008 em 20,8\% e Guedes et al. 2011 em $34,6 \%$ em populações brasileiras. A discrepância entre os resultados observados pode estar relacionada às diferentes metodologias e a variabilidade anatômica existente entre diferentes grupos populacionais.

A classe $\mathrm{V}$, onde forame está localizado entre o segundo pré-molar e primeiro molar, foi observada em $10,5 \%$ dos casos (Tabela 2). A presença do FM posicionado à baixo dos primeiros molares é rara, classe VI. No presente estudo, esta posição ocorreu em $2 \%$ da amostra, Ngeow, Yuzawati (2003) observaram somente em $0,98 \%$.

Em apenas $1 \%$ da amostra, observou-se forame mentual com padrão de classificação I, entre o canino e o pré-molar (Tabela 2). Resultado semelhante foi encontrado por Ngeow, Yuzawati (2003) que verificaram em 0,98\%; enquanto Filho et al., (2011) e Neiva et al. (2004) não observaram FM nesta posição. A localização abaixo do primeiro pré-molar, classe II, esteve presente em $2 \%$ observados no antímero esquerdo, sendo todas do gênero feminino, sem justificativa aparente.

As maiores alturas do forame mentual, em relação à base da mandíbula do lado esquerdo, foram $17 \mathrm{~mm}$ e do lado direito $19 \mathrm{~mm}$. Levando-se em consideração a ampliação da radiografia panorâmica de $25 \%$, o resultado foi de $13 \mathrm{~mm}$ e $14 \mathrm{~mm}$, respectivamente. Para classe III, a média das alturas foi $12 \mathrm{~mm}$, classe IV $13 \mathrm{~mm}$, classe V 12 mm e classe VI $12 \mathrm{~mm}$ e classe I $10 \mathrm{~mm}$ para ambos os antímeros. Classe II não foi observada no antímero direito (Tabelas 3 e 4).

Analisando-se as variáveis, nos antímeros esquerdo e direito, constatou-se relativa proximidade entre as dimensões e a localização do forame mentual nos dois lados de um mesmo exame radiográfico. Assim, infere-se 


\begin{tabular}{llll}
\hline \multirow{2}{*}{ Classificação de Tebo, Telford } & \multicolumn{2}{c}{ Antímero direito } \\
\hline & & Média & Desvio padrão \\
\cline { 2 - 4 } Forame esquerdo & Tipo I & 0,10 & X \\
\cline { 2 - 4 } & Tipo II & 0,12 & 0,01 \\
\cline { 2 - 4 } & Tipo III & 0,12 & 0,02 \\
\cline { 2 - 4 } & Tipo IV & 0,13 & 0,02 \\
\cline { 2 - 4 } & Tipo V & 0,12 & 0,03 \\
\hline
\end{tabular}

Tabela 3. Média em milímetros da altura do forame mentual no antímero esquerdo em relação à base da mandíbula seguindo a classificação proposta por Tebo e Telford (1950). Fonte: Acervo de Radiografias do UNINOVAFAPI, Teresina-PI.

\begin{tabular}{llcc}
\hline \multirow{2}{*}{ Classificação de Tebo, Telford } & \multicolumn{2}{c}{ Antímero direito } \\
\hline & & Média & Desvio padrão \\
\cline { 2 - 4 } & & Tipo I & 0,10 \\
Forame direito & Tipo II & $X$ & X \\
\cline { 2 - 4 } & Tipo III & 0,12 & 0,02 \\
\cline { 2 - 4 } & Tipo IV & 0,13 & 0,02 \\
\cline { 2 - 4 } & Tipo V & 0,12 & 0,01 \\
\cline { 2 - 4 } & Tipo VI & 0,12 & 0,03 \\
\hline
\end{tabular}

Tabela 4. Média em milímetros da altura do forame mentual no antímero direito em relação à base da mandíbula seguindo a classificação proposta por Tebo e Telford (1950). Fonte: Acervo de Radiografias do UNINOVAFAPI, Teresina-PI.

que a radiografia panorâmica fornece referências topográficas do forame mentual aos Cirurgiões-dentistas na prática clínica.

\section{CONCLUSÃO}

Há variações na localização do forame mentual. A maior prevalência observada foi de forames localizados entre os primeiros e segundos pré-molares inferiores, classe III, com distância à base da mandíbula de $12 \mathrm{~mm}$ em ambos os antímeros. Porém há proximidade entre as dimensões e a localização do forame mentual nos dois lados de um mesmo exame radiográfico.

\section{REFERÊNCIAS}

ANDRADE, Y.D.N.; ARAUJO, E.B.J.; SOUZA, L.M.A.; GROPPO, F.C. Análise das variações anatômicas do canal da mandíbula encontradas em radiografias panorâmicas. Rev Odontol UNESP, v. 44, n.1, p. 31-36, 2015.

APINHASMIT. W; METHATHRATHIP, D CHOMPOOPONG, S; SANGVICHIEN, S. Mental foramen in Thais: an anatomical variation related to gender and side. Surg Radiol Anat. v.28, p.529-33, 2006.
ARAÚJO, J. A. D; TEIXEIRA, F.R; SILVEIRA, E.M; ROCHA, C.R.C; MIRANDA, J. L. Estudo radiográfico quanto à variação da posição anatômica do forame mentoniano. Arquivos em Odontologia, Belo Horizonte, v. 14, n.2, p. 105-192, 2005.

BAHLIS, A; MEZZOMO, L.A.; BOECKEL, D; COSTA, N. P; TEIXEIRA, E.Rl. Accuracy of periapical radiography, panoramic radiography and computed tomography for examining the mental foramen region. Rev. Odonto Ciênc.; v. 25, n.3, p. 282-287, 2010.

COMANDULli. F; DINATO, J.C; DUTRA, V; SUSIN, C. Correlation between panoramic radiograph and computed tomography measurements for the evaluation of vertical bone height in oral implantology. Cienc Odontol Bras. v.8, n.2, p. 54$59,2005$.

FILHO, L.R.A; REIS, H.S.M; AMADEI, S.U; SCHERMA, A.P; SOUZA, D.M. Avaliação da posição do forame mentual em Relação aos dentes e base da mandíbula na Radiografia panorâmica convencional. Braz J Periodontol, v.21, 2011.

GUEDES, O.A; RABELO L.E.G; PORTO, O.C.L; ALENCAR, A.H.G; ESTRELA, C. Avaliação radiográfica da posição e forma do forame mentual em uma subpopulação Brasileira. Rev Odontol Bras Central: v. 20, n. 53, p. 160-165, 2011.

GUO, J.L; SU, L; ZHAO, J.L; YANG, L; LV D. L.; LI, Y.Q. Location of mental foramen based on soft- and hard-tissue 
landmarks in a Chinese population. J Craniofac Surg. v, 20, n. 6, p. 2235-2237, 2009.

HAGHANIFAR. S; ROKOUEI, M. Radiographic evaluation of the mental foramen in a selected Iranian population. Indian J Dent Res. v. 20, n.2, p. 150-2, 2009.

KIM, I.S; KIM, S.G; KIM, Y.K; KIM, J.D. Position of the mental foramen in a Korean population: a clinical and radiographic study. Implant Dent. v.15, n.4, p.404-411, 2006.

LEITE, A.F; FIGUEIREDO, P.T.F; GUIA, C.M; MELO, N.S PAULA, A.P. Panoramic Radiograph - Auxiliary Tool in the Diagnosis of Osteoporosis. Rev Bras Reumatol. v.48, n.4, p. 226-233, 2008.

LIMA D.S.C. FIGUERÊDO, A.A; GRAVINA, P.R; MENDONÇA, V.R.R; CASTRO, M.P; CHAGAS, G.L; ARAÚJO, L.P; SILVA, V.H.M.V.C; ROCHA, E.A; LIMA, J.V. Caracterização anatômica do forame mentual em uma amostra de mandíbulas humanas secas brasileiras. Rev Bras Cir Craniomaxilofac; v13, n4, p. 230-235, 2010.

MORAES, M.E.L; MANHÃES JUNIOR, L. R. C; MORAES, L. C; MEDICI FILHO, E. CASTILHO, J.C.M; VAROLI, F.P; XAVIER, J. Localização vertical e horizontal em relação ao segundo pré-molar inferior pelo método radiográfico. RGO, Porto Alegre, v. 56, n.1, p. 47-52, 2008.

MANHÃES JR, L.R.C, MORAES M. E. L.; MORAES, L. C; MÉDICI FILHO, E; CASTILHO, J. C. M. Classificação do forame mentoniano e sua correlação bilateral Revista Odonto, v. 17, n. 33, 2009.

NGEOW, W.C; YUZAWATI, Y. The location of the mental foramen in a selected, Malay population. J Oral Sci, v.45, n.3, p.171-175, 2003.

NEIVA, R.F.; GAPSKS, R.; WANG, H. L. Morphometric Analysis of Implant-Related Anatomy in Caucasian Skulls. J. Periodontal, v.75, n.8, p.1061-1067, 2004.

NEVES, F.S, OLIVEIRA, L.S.A.F, TORRES, M.G.G, CRUSÓE-SOUSA, M, OLIVEIRA C, CAMPOS P.S.F, CRUSÓE-RABELLO I. Accessory mental foramen: case report. Rev Pós Grad, v.17, n.3, p.173-176, 2010.

RIBEIRO, I.L.A; FERREIRA, M.G.R. Differential diagnosis of periapical lesion suggestive image. Revista Cubana de Estomatología, v.52, n1, p.96-102, 2015.

SAITO, K; ARAÚJO, N.S; SAITO, M.T; PINHEIRO, J.J.V.P; CARVALHO, P.L. Analysis of the mental foramen using cone beam computerized tomography. Rev Odontol UNESP. v.44, n.4, p. 226-231, 2015.

SANKAR, D. K; BHANU, S. P; SUSAN, P. J. Morphometrical and morphological study of mental foramen in dry dentulous mandibles of South Andhra population of India. Indian Journal of Dental Research, v. 22, n.4, p. 542-546, 2011.

SALVADOR, J.F; MEDEIROS, P.L; JUNIOR, O.F; CAPELOZZA, A.L.A. Radiographic anatomy of the mandibular canal and its variations in panoramic radiographs. Innov Implant J, Biomater Esthet. v.5, n.2, p. 19-24, 2010.
SHAH, S; VAZE, S; KRITI K. A Variation in the Position of the Mental Foramen: A Case Report. J Maxillofac Oral Surg, v. 9, n. 3, p. 307-309, 2010.

TEBO, H. G; TELFORD, I. R. An analysis of the variations in position of the mental foramen. Anat Rec, v.107, n.1, p.61-6, 1950.

TEIXERA, R. C. et al. Prevalência da Localização do Forame Mentual em Radiografias Periapicais. Revista da ABRO, v. 8, n. 1, p. 16-21, 2007. 